\title{
One-Pot Synthesis of 2,4,5-Triphenyl Imidazoles from 1,2-Diols as Key Reagents
}

\author{
Narasashetty Jagadishbabu and Kalegowda Shivashankar* \\ P. G. Department of Chemistry, Central College Campus, Bangalore University, Bangalore 560001 , India
}

(Received: September 16, 2016; Accepted: February 2, 2017; DOI: 10.1002/jccs.201600746)

\begin{abstract}
A simple one-pot procedure for the preparation of 2,4,5-triphenyl imidazole derivatives is presented. The procedure involves the lead tetraacetate oxidation of 1,2-diols to give aldehydes in situ, which then undergo a three-component reaction with benzil and ammonium acetate to yield the imidazole derivatives.
\end{abstract}

Keywords: Imidazoles; Lead tetraacetate; Multicomponent reaction; 1,2-Diols; Benzil; Ammonium acetate.

\section{INTRODUCTION}

Multicomponent reactions (MCRs) have emerged as a powerful tool for industrial and academic research groups because of their shorter reaction times as well as the avoidance of the isolation of intermediates, their purification, and characterization. ${ }^{1-3}$ Imidazoles have a privileged structure as they occur as fragments in drugs displaying a wide spectrum of pharmacological activities. $^{4-9}$ A large number of compounds bearing substituted imidazole derivatives have entered preclinical and clinical trials over the last few years. These derivatives represent an important structural motif in commercial drugs ${ }^{10}$ such as the anti-gastroesophageal reflux drug Omeprazole, platelet aggregation inhibitor Trifenagrel, angiotensin II receptor blocker Olmesartan, and the anti-high-blood-pressure drugs Eprosartan and Losartan (Figure 1).

Imidazole derivatives have received significant importance because of their diverse biological properties such as anti-inflammatory, ${ }^{11}$ antimicrobial, ${ }^{12}$ anticancer, ${ }^{13}$ antitubercular, ${ }^{14}$ antifungal, ${ }^{15}$ antibacterial, ${ }^{16}$ antiviral, ${ }^{17}$ antioxidant, ${ }^{18}$ and amebicidal activities. ${ }^{19}$ The great potential of substituted imidazole derivatives in the pharmaceuticals field has therefore triggered growing interest in their synthetic study.

These applications have stimulated widespread interest in the synthesis of imidazole derivatives. The typical syntheses of imidazole derivatives have been well documented in the literature. ${ }^{20-22}$ The most important of these are (1) one-pot cyclocondensation of aldehydes with benzoin or benzil and ammonium acetate, ${ }^{23}$ (2) one-pot cyclocondensation of aldehydes with benzil, aromatic amine, and ammonium acetate, ${ }^{24}$ and (3) one-pot cyclocondensation of aromatic nitriles. ${ }^{25}$ This has led to the development of new, improved methodologies involving the use of a number of catalysts such as $\mathrm{Yb}(\mathrm{OPf})_{3},{ }^{26} \mathrm{Cu}\left(\mathrm{NO}_{3}\right)_{2}$ /zeolite, ${ }^{27}$ potassium dihydrogen phosphate, ${ }^{28} \mathrm{ZrOCl}_{2} \cdot 8 \mathrm{H}_{2} \mathrm{O},{ }^{29}$ $\mathrm{Zr}$ (acac) $)_{4}{ }^{30} \quad \mathrm{NiCl}_{2} \cdot 6 \mathrm{H}_{2} \mathrm{O},{ }^{31} \quad p$-toluenesulfonic acid (PTSA), ${ }^{32} \mathrm{TiCl}_{4}-\mathrm{SiO}_{2},{ }^{33} \mathrm{MCM}-41,{ }^{34} \mathrm{ZrCl}_{4},{ }^{35}$ sodium bisulfite, ${ }^{36}$ polymer-supported zinc chloride, ${ }^{37}$ alum, ${ }^{38}$ zinc oxide, ${ }^{39}$ trichloroisocyanuric acid, ${ }^{40}$ tetrabutyl ammonium bromide, ${ }^{41}$ ceric ammonium nitrate, ${ }^{42}$ nano copper/cobalt ferrites, ${ }^{43}$ microwave, ${ }^{44}$ amberlyst, ${ }^{45}$ novel polymers, ${ }^{46}$ antimony trichloride/stannous chloride dihydrate, ${ }^{47} \mathrm{SbCl}_{3} / \mathrm{SiO}_{2},{ }^{48}$ sulfamic acid/ $/ \mathrm{Fe}_{3} \mathrm{O}_{4},{ }^{49}$ and nano aluminum nitride. ${ }^{50}$ Although several methodologies have been documented, most of them have their own drawbacks such as poor yields, the use of toxic catalysts, and tedious work-up. Aldehydes are ubiquitous substrates in many powerful MCRs. However, they are in general unstable, especially because of aerial oxidation to acids, and are prone to polymerization or hydrolysis. Thus, in many cases aldehydes must be purified just before their use because the presence of other products not only affects the concentration of the active aldehyde but also interferes with the chemical reactions. ${ }^{51}$ Hence, there is a need to develop a rapid and efficient synthetic protocol for the one-pot synthesis of substituted imidazole scaffolds.

*Corresponding author. Email: shivashankark@gmail.com 

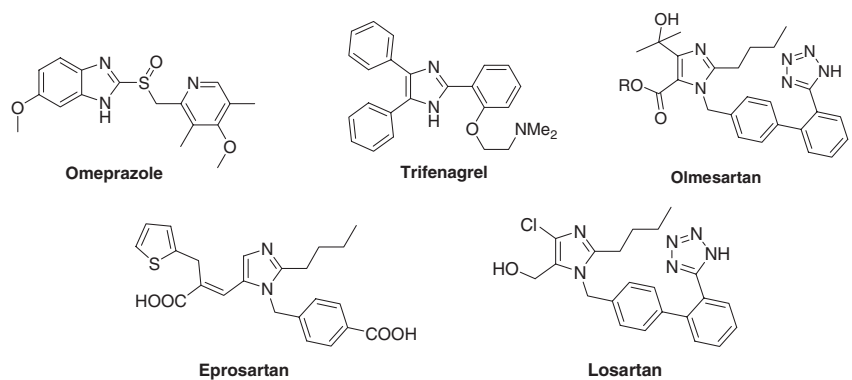

Fig. 1. Typical imidazole drugs.

In continuation of our work on the synthesis of heterocycles and development of useful synthetic methodologies, ${ }^{2,52-57}$ in this study we report the use of lead tetraacetate as an oxidizing agent for the synthesis of 2,4,5-triphenyl imidazole derivatives under mild conditions. Lead tetraacetate is an inexpensive, commercially available, and easy-to-handle reagent.

\section{RESULTS AND DISCUSSION}

In our preliminary studies, 1 equiv of 1,2-diphenyl-1,2-ethanediol 1a, 2 equiv of benzil 2, 4 equiv of ammonium acetate $\mathbf{3}$, and 1 equiv of lead tetraacetate in dioxane were chosen for the model reaction (Scheme 1). Initially, when the model reaction was carried without lead tetraacetate from room temperature to $101^{\circ} \mathrm{C}$, no desirable product was observed even after prolonged reaction time. This indicated that an oxidizing agent was absolutely necessary for the reaction. Interestingly, in the presence of lead tetraacetate, when the same set of substrates were tested under reflux condition in dioxane, they provided $4 \mathrm{a}$ in $71 \%$ yield in $4.5 \mathrm{~h}$. The compound $4 \mathbf{a}$ was confirmed by IR and ${ }^{1} \mathrm{H}$ NMR. From this encouraging result, we were prompted to check whether the yield could be further improved by changing the solvent. Other solvents such as DCM, acetonitrile, ethanol, acetone, THF, methanol, DMF, and DMSO were screened (Table 1) under reflux condition for the
Table 1. Optimization of solvents for the synthesis (4a)

\begin{tabular}{llll}
\hline Entry & Solvents & Time (h) & Yield (\%) \\
\hline 1 & DCM & 5.5 & 48 \\
2 & Dioxane & 4.5 & 71 \\
3 & Acetonitrile & 3 & 90 \\
$\mathbf{4}$ & Ethanol & $\mathbf{3}$ & $\mathbf{9 6}$ \\
5 & Acetone & 4.5 & 74 \\
6 & THF & 4 & 76 \\
7 & Methanol & 3.5 & 84 \\
8 & DMF & 5.5 & 45 \\
9 & DMSO & 5.5 & 52 \\
\hline
\end{tabular}

same reaction. Among the solvents, ethanol was found to be the best one for this reaction in terms of yield and reaction time.

The scope and limitations of this three-component reaction under optimized reaction conditions were studied using a variety of 1,2-diols, benzil, and ammonium acetate. The reaction worked well in case of 1,2-diols tethered with $\mathrm{OH}, \mathrm{Cl}, \mathrm{Br}, \mathrm{F}$, and $\mathrm{OCH}_{3}$ groups. Moderate yield was obtained for the reaction involving 1,2diol tethered with $\mathrm{NO}_{2}$ group. In all cases, the reaction proceeded smoothly to afford the desired products with good to excellent yields (Table 2).

Mechanistically, ${ }^{60,61}$ lead tetraacetate oxidizes 1,2diol to benzaldehyde giving acetic acid, which facilitates the formation of the imine between benzaldehyde and ammonium acetate. The imine then reacts with the intermediate that is formed by the reaction of benzil with ammonium acetate to give the corresponding imidazole via cyclization and dehydration (Figure 2).

\section{EXPERIMENTAL}

\section{General information}

Melting points were determined on an electric melting point apparatus and were uncorrected. Fourier transform infrared (FT-IR) spectra were recorded on an Agilent Cary 630 instrument. ${ }^{1} \mathrm{H}$ NMR (400 MHz)

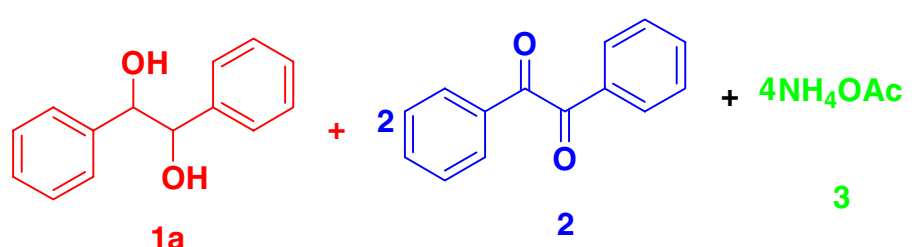

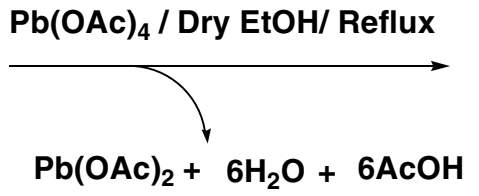

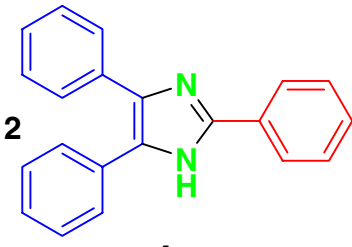

$4 a$

Scheme 1 Synthesis of 2,4,5-triphenyl imidazole derivatives using lead tetraacetate as a reagent. 
Table 2. Synthesis of 2,4,5-triphenyl imidazole derivatives (4a-o)

\begin{tabular}{|c|c|c|c|c|c|c|}
\hline Entry & 1,2-Diols & Products & Time (h) & Yield (\%) & Obs. $\mathrm{Mp}\left({ }^{\circ} \mathrm{C}\right)$ & Lit. $\mathrm{Mp}\left({ }^{\circ} \mathrm{C}\right)^{\mathrm{ref}}$ \\
\hline 1 & $1 a$ & $4 a$ & 3 & 96 & $276-278$ & $275-277^{43}$ \\
\hline 2 & $1 b$ & $4 \mathrm{~b}$ & 2.5 & 89 & $233-235$ & $234-236^{39}$ \\
\hline 3 & & & 2.5 & 90 & $244-246$ & $245-247^{38}$ \\
\hline 4 & $1 d$ & $4 d$ & 3 & 91 & $218-220$ & $218-220^{58}$ \\
\hline 5 & & & 3.5 & 92 & $231-233$ & \\
\hline 6 & & $4 f$ & 4.5 & 82 & $242-244$ & $240-242^{23}$ \\
\hline 7 & & & 4 & 89 & $255-257$ & \\
\hline 8 & & $4 h$ & 3 & 92 & $232-234$ & $233-235^{39}$ \\
\hline 9 & $1 \mathrm{i}$ & $4 \mathbf{i}$ & 3.5 & 91 & $246-248$ & $248-250^{39}$ \\
\hline
\end{tabular}


Table 2. Continued

\begin{tabular}{|c|c|c|c|c|c|c|}
\hline Entry & 1,2-Diols & Products & Time (h) & Yield (\%) & Obs. Mp $\left({ }^{\circ} \mathrm{C}\right)$ & Lit. $\mathrm{Mp}\left({ }^{\circ} \mathrm{C}\right)^{\mathrm{ref}}$ \\
\hline 10 & & & 3 & 90 & $238-240$ & $239-241^{23}$ \\
\hline 11 & & & 4 & 89 & $257-259$ & \\
\hline 12 & & & 2.5 & 95 & $255-257$ & $256-258^{54}$ \\
\hline 13 & & & 4 & 88 & $263-265$ & $265-267^{59}$ \\
\hline 14 & & & 2 & 96 & 219-221 & $218-220^{36}$ \\
\hline 15 & & & 4 & 89 & $263-265$ & \\
\hline
\end{tabular}

and ${ }^{13} \mathrm{C}$ NMR (100 MHz) spectra were recorded in $\mathrm{CDCl}_{3}$ and DMSO- $d_{6}$ with TMS as internal standard using a Bruker spectrometer. Chemical shifts are expressed in $\delta$ ppm. Elemental analyses were carried out using an Elemental Vario Micro Cube Rapid Analyzer. Lead tetraacetate is a solid compound, and its purity is $\geq 99.99 \%$.

\section{Typical experimental procedure for the synthesis of}

\section{2,4,5-triphenyl-1 $H$-imidazole (4a)}

Lead tetraacetate $(0.44 \mathrm{~g}, 1 \mathrm{mmol})$ was added to a solution of 1,2-diphenylethane-1,2-diol $(0.21 \mathrm{~g}, 1 \mathrm{mmol})$ in dry ethanol $(10 \mathrm{~mL})$. The reaction mixture was stirred at room temperature for $5 \mathrm{~min}$. Then, benzil ( $0.42 \mathrm{~g}, 2 \mathrm{mmol})$ and ammonium acetate $(0.32 \mathrm{~g}, 4.2 \mathrm{mmol})$ were added to a round-bottom flask fitted with a reflux condenser and a guard tube. Then reaction mixture was heated at $70^{\circ} \mathrm{C}$ in an oil bath for $3 \mathrm{~h}$. The reaction was allowed to cool to room temperature, and $10 \mathrm{~mL}$ of water was added. The resulting precipitate was collected by filtration and washed with cold ethanol to afford the product. The crude product was purified by recrystallization from ethanol.

\section{CONCLUSION}

In conclusion, we have developed an efficient and facile method for the synthesis of 2,4,5-triphenyl 
Step 1.

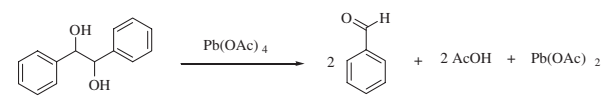

Step 2.
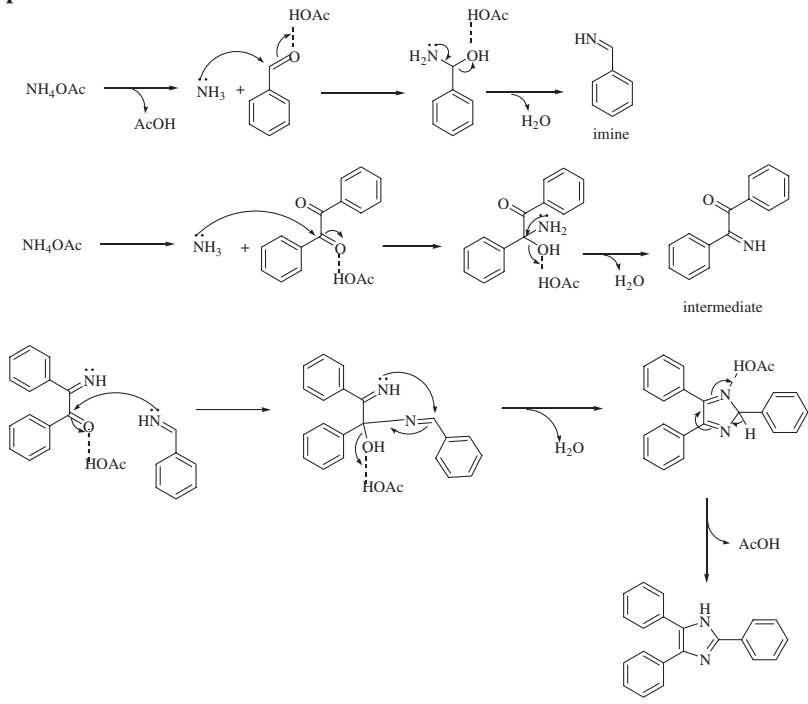

Fig. 2 Plausible mechanism for the formation of imidazole derivatives.

imidazole derivatives via a one-pot, three-component condensation reaction between 1,2-diols, benzil, and ammonium acetate. The current strategy can be used to decrease the number of steps in a multistep synthesis.

\section{Spectral data of imidazole compounds}

2,4,5-Triphenyl- $\mathbf{H}$-imidazole (4a). A pale brown solid, $284 \mathrm{mg}\left(96 \%\right.$ yield). IR (ATR, $\left.\mathrm{cm}^{-1}\right)$ : $3325(\mathrm{NH})$; mp $275-277^{\circ} \mathrm{C} .{ }^{1} \mathrm{H}$ NMR (DMSO- $d_{6}, 400 \mathrm{MHz}$ ): $\delta$ $9.22(\mathrm{~s}, 1 \mathrm{H}, \mathrm{NH}), 8.05-6.73(\mathrm{~m}, 15 \mathrm{H}$, Ar-H) ppm. Anal. Calcd for $\mathrm{C}_{21} \mathrm{H}_{16} \mathrm{~N}_{2}$ : C, 85.11; H, 5.44; N, 9.45; found: $\mathrm{C}, 85.09 ; \mathrm{H}, 5.43 ; \mathrm{N}, 9.42 \%$.

2-(4-Chlorophenyl)-4,5-diphenyl-1 $H$-imidazole (4b). A white solid, $293 \mathrm{mg}$ ( $89 \%$ yield). IR (ATR, $\mathrm{cm}^{-1}$ ): $3328(\mathrm{NH})$; mp 234-236 ${ }^{\circ} \mathrm{C} .{ }^{1} \mathrm{H}$ NMR (DMSO- $d_{6}$, $400 \mathrm{MHz}): \delta 12.79(\mathrm{~s}, 1 \mathrm{H}, \mathrm{NH}), 8.15-7.21(\mathrm{~m}, 14 \mathrm{H}$, Ar-H) ppm. Anal. Calcd for $\mathrm{C}_{21} \mathrm{H}_{15} \mathrm{~N}_{2} \mathrm{Cl}$ : C, 76.24; $\mathrm{H}$, 4.57; N, 8.47; found: C, 76.22; H, 4.56; N, 8.44\%.

2-(3-Chlorophenyl)-4,5-diphenyl-1 $H$-imidazole (4c). A white solid, $297 \mathrm{mg}$ (90\% yield). IR (ATR, $\left.\mathrm{cm}^{-1}\right)$ : $3315(\mathrm{NH}) ; \quad \mathrm{mp} \quad 245-247^{\circ} \mathrm{C} .{ }^{1} \mathrm{H}$ NMR $\left(\mathrm{CDCl}_{3}\right.$, $400 \mathrm{MHz}): \delta 11.25(\mathrm{~s}, 1 \mathrm{H}, \mathrm{NH}), 8.40-7.24(\mathrm{~m}, 14 \mathrm{H}$, Ar-H) ppm. Anal. Calcd for $\mathrm{C}_{21} \mathrm{H}_{15} \mathrm{~N}_{2} \mathrm{Cl}$ : C, 76.24; $\mathrm{H}$, 4.57; N, 8.47; found: C, 76.22; H, 4.56; N, 8.44\%.
2-(4-Ethoxyphenyl)-4,5-diphenyl- $1 \mathrm{H}$-imidazole (4d). A pale brown solid, $309 \mathrm{mg}$ ( $91 \%$ yield). IR (ATR, $\mathrm{cm}^{-1}$ ): $3330(\mathrm{NH}) ; \mathrm{mp} 218-220^{\circ} \mathrm{C} .{ }^{1} \mathrm{H}$ NMR $\left(\mathrm{CDCl}_{3}\right.$, $400 \mathrm{MHz}): \delta 10.03$ (s, 1H, NH), 8.41-6.84 (m, 13H, Ar$\mathrm{H}), 3.98\left(\mathrm{~m}, 2 \mathrm{H}, \mathrm{CH}_{2}\right), 1.39\left(\mathrm{~m}, 3 \mathrm{H}, \mathrm{CH}_{3}\right) \mathrm{ppm}$. Anal. Calcd for $\mathrm{C}_{23} \mathrm{H}_{20} \mathrm{~N}_{2} \mathrm{O}$ : C, 81.15; H, 5.92; N, 8.23; found: C, $81.12 ; \mathrm{H}, 5.90 ; \mathrm{N}, 8.21 \%$.

2-(3-Bromo-4-methoxyphenyl)-4,5-diphenyl- $1 \mathrm{H}$-imidazole (4e). A pale brown solid, $372 \mathrm{mg}$ (92\% yield). IR $\left(\right.$ ATR, $\left.\mathrm{cm}^{-1}\right)$ : $3320(\mathrm{NH})$; mp 231-233 ${ }^{\circ} \mathrm{C} .{ }^{1} \mathrm{H}$ NMR (DMSO- $\left.d_{6}, 400 \mathrm{MHz}\right): \delta 12.59\left(\mathrm{~s}, 1 \mathrm{H}, \quad \mathrm{NH}, \mathrm{D}_{2} \mathrm{O}\right.$ exchangeable), 8.30-7.19 (m, 13H, Ar-H), 3.90 (s, 3H, $\left.\mathrm{OCH}_{3}\right)$ ppm. ${ }^{13} \mathrm{C}$ NMR (DMSO- $\left.d_{6}, 100 \mathrm{MHz}\right): \delta$ $155.3,144.0,136.9,135.0,130.9,129.4,128.5,128.2$, $128.1,128.0,127.6,127.0,126.4,125.8,124.4,112.8$, 110.8, 56.3 ppm. Anal. Calcd for $\mathrm{C}_{22} \mathrm{H}_{17} \mathrm{~N}_{2} \mathrm{OBr}$ : C, 65.20; H, 4.23; N, 6.91; found: $\mathrm{C}, 65.18 ; \mathrm{H}$, $4.20 ; \mathrm{N}, 6.88 \%$.

2-(4-Nitrophenyl)-4,5-diphenyl-1 $H$-imidazole (4f). A brown solid, $279 \mathrm{mg}$ ( $82 \%$ yield). IR (ATR, $\left.\mathrm{cm}^{-1}\right)$ : $3320(\mathrm{NH}) ; \mathrm{mp} \quad 240-242^{\circ} \mathrm{C} .{ }^{1} \mathrm{H}$ NMR $\left(\mathrm{CDCl}_{3}\right.$, $400 \mathrm{MHz}): \delta 10.02(\mathrm{~s}, 1 \mathrm{H}, \mathrm{NH}), 8.26-7.26(\mathrm{~m}, 14 \mathrm{H}$, Ar-H) ppm. Anal. Calcd for $\mathrm{C}_{21} \mathrm{H}_{15} \mathrm{~N}_{3} \mathrm{O}_{2}: \mathrm{C}, 73.89 ; \mathrm{H}$, 4.43; N, 12.31; found: C, 73.88; H, 4.41; N, 12.28\%.

2-(3-Bromo-4-chlorophenyl)-4,5-diphenyl-1 H-imidazole (4g). A white solid, $364 \mathrm{mg}$ (89\% yield). IR (ATR, $\mathrm{cm}^{-1}$ ): $3335(\mathrm{NH})$; mp 255-257 ${ }^{\circ} \mathrm{C} .{ }^{1} \mathrm{H}$ NMR (DMSO$\left.d_{6}, 400 \mathrm{MHz}\right): \delta 12.77(\mathrm{~s}, 1 \mathrm{H}, \mathrm{NH}), 8.41-7.21(\mathrm{~m}, 13 \mathrm{H}$, Ar-H) ppm. ${ }^{13} \mathrm{C}$ NMR (DMSO- $d_{6}, 100 \mathrm{MHz}$ ): $\delta 159.2$, $156.8,143.1,137.3,134.8,130.7,129.7,128.6,128.4$ (2C), 128.2, 128.1, 127.8, 127.0, 126.6, 126.4, 126.3, 117.2, 117.0, 108.5, $108.2 \mathrm{ppm}$. Anal. Calcd for $\mathrm{C}_{21} \mathrm{H}_{14} \mathrm{~N}_{2} \mathrm{BrCl}$ : C, 61.56; H, 3.44; $\mathrm{N}, 6.84$; found: $\mathrm{C}$, $61.54 ; \mathrm{H}, 3.43 ; \mathrm{N}, 6.82 \%$.

4,5-Diphenyl-2-p-tolyl-1 $\boldsymbol{H}$-imidazole (4h). A white solid, $285 \mathrm{mg}\left(92 \%\right.$ yield). IR (ATR, $\left.\mathrm{cm}^{-1}\right): 3312(\mathrm{NH})$; mp $233-235^{\circ} \mathrm{C} .{ }^{1} \mathrm{H}$ NMR (DMSO- $d_{6}, 400 \mathrm{MHz}$ ): $\delta$ $12.56(\mathrm{~s}, 1 \mathrm{H}, \mathrm{NH}), 7.98-7.19(\mathrm{~m}, 14 \mathrm{H}, \operatorname{Ar}-\mathrm{H}), 2.35$ (s, $\left.3 \mathrm{H}, \mathrm{CH}_{3}\right)$ ppm. Anal. Calcd for $\mathrm{C}_{22} \mathrm{H}_{18} \mathrm{~N}_{2}$ : C, 85.13; H, 5.85; N, 9.03; found: $\mathrm{C}, 85.11 ; \mathrm{H}$, $5.82 ; \mathrm{N}, 9.02 \%$.

2-(4-Bromophenyl)-4,5-diphenyl-1 $\boldsymbol{H}$-imidazole (4i). A white solid, $341 \mathrm{mg}$ ( $91 \%$ yield). IR (ATR, $\mathrm{cm}^{-1}$ ): $3320(\mathrm{NH}) ; \mathrm{mp} 248-250^{\circ} \mathrm{C} .{ }^{1} \mathrm{H}$ NMR (DMSO- $d_{6}$, $400 \mathrm{MHz}): \delta 12.61(\mathrm{~s}, 1 \mathrm{H}, \mathrm{NH}), 7.79-7.22(\mathrm{~m}, 14 \mathrm{H}$, Ar-H) ppm. Anal. Calcd for $\mathrm{C}_{21} \mathrm{H}_{15} \mathrm{~N}_{2} \mathrm{Br}$ : C, 67.21; $\mathrm{H}$, 4.03; N, 7.47; found: C, 67.18; H, 4.02; N, 7.45\%. 
2-(4-Fluorophenyl)-4,5-diphenyl-1H-imidazole (4j). A pale brown solid, $282 \mathrm{mg}(90 \%$ yield). IR (ATR, $\mathrm{cm}^{-1}$ ): $3322(\mathrm{NH})$; mp $239-241{ }^{\circ} \mathrm{C} .{ }^{1} \mathrm{H}$ NMR (DMSO$d_{6}, 400 \mathrm{MHz}$ ): $\delta 12.80(\mathrm{~s}, 1 \mathrm{H}, \mathrm{NH}), 7.60-7.23(\mathrm{~m}, 12 \mathrm{H}$, Ar-H) ppm. Anal. Calcd for $\mathrm{C}_{21} \mathrm{H}_{15} \mathrm{~N}_{2} \mathrm{~F}: \mathrm{C}, 80.24$; $\mathrm{H}$, 4.81 ; N, 8.91; found: C, 80.22; H, 4.78; N, 8.90\%.

2-(4-Bromo-3-chlorophenyl)-4,5-diphenyl-1 H-imidazole (4k). A white solid, $364 \mathrm{mg}$ (89\% yield). IR (ATR, $\mathrm{cm}^{-1}$ ): 3315 (NH); mp $257-259^{\circ} \mathrm{C} .{ }^{1} \mathrm{H}$ NMR (DMSO$\left.d_{6}, 400 \mathrm{MHz}\right): \delta 12.76$ (s, 1H, NH), 8.27-7.22 (m, 13H, Ar-H) ppm. ${ }^{13} \mathrm{C}$ NMR (DMSO- $d_{6}, 100 \mathrm{MHz}$ ): $\delta$ 158.2, $155.7,143.2,137.3,134.8,130.7,128.6,128.2,128.1$ (2C), 127.8, 127.0, 126.8, 126.6, 125.7 (2C), 120.0, 119.8, 117.4, $117.2 \mathrm{ppm}$. Anal. Calcd for $\mathrm{C}_{21} \mathrm{H}_{14} \mathrm{~N}_{2} \mathrm{ClBr}$ : C, 61.56; H, 3.44; $\mathrm{N}, 6.84$; found: $\mathrm{C}, 61.53 ; \mathrm{H}$, $3.42 ; \mathrm{N}, 6.83 \%$.

4-(4,5-Diphenyl-1 $H$-imidazol-2-yl)phenol (4I). A white solid, $296 \mathrm{mg}$ (95\% yield). IR (ATR, $\mathrm{cm}^{-1}$ ): 3324 (NH); $m p \quad 256-258^{\circ} \mathrm{C} .{ }^{1} \mathrm{H}$ NMR (DMSO- $d_{6}$, $400 \mathrm{MHz}$ ): $\delta 12.58(\mathrm{~s}, 1 \mathrm{H}, \mathrm{NH}), 9.51(\mathrm{~s}, 1 \mathrm{H}, \mathrm{OH})$, 7.54-6.76 (m, 14H, Ar-H) ppm. Anal. Calcd for $\mathrm{C}_{21} \mathrm{H}_{16} \mathrm{~N}_{2} \mathrm{O}$ : C, 80.75; $\mathrm{H}, 5.16$; $\mathrm{N}, 8.97$; found: $\mathrm{C}$, $80.72 ; \mathrm{H}, 5.14 ; \mathrm{N}, 8.96 \%$.

\section{2-(2,6-Dichlorophenyl)-4,5-diphenyl- $\mathbf{H}$-imidazole}

(4m). A white solid, $321 \mathrm{mg}$ (88\% yield). IR (ATR, $\mathrm{cm}^{-1}$ ): $3310(\mathrm{NH})$; mp $265-267^{\circ} \mathrm{C} .{ }^{1} \mathrm{H}$ NMR (DMSO$\left.d_{6}, 400 \mathrm{MHz}\right): \delta 12.72(\mathrm{~s}, 1 \mathrm{H}, \mathrm{NH}), 7.65-7.20(\mathrm{~m}, 13 \mathrm{H}$, Ar-H) ppm. Anal. Calcd for $\mathrm{C}_{21} \mathrm{H}_{14} \mathrm{~N}_{2} \mathrm{Cl}_{2}: \mathrm{C}, 69.05 ; \mathrm{H}$, 3.86; N, 7.67; found: C, 69.03; H, 3.83; N, 7.66\%.

\section{2-(3,4-Dimethoxyphenyl)-4,5-diphenyl-1 $\mathrm{H}$-imidazo-} le (4n). A white solid, $341 \mathrm{mg}$ ( $96 \%$ yield). IR (ATR, $\mathrm{cm}^{-1}$ ): $3332(\mathrm{NH}) ; \mathrm{mp} 218-220^{\circ} \mathrm{C} .{ }^{1} \mathrm{H}$ NMR (DMSO$\left.d_{6}, 400 \mathrm{MHz}\right): \delta 12.01(\mathrm{~s}, 1 \mathrm{H}, \mathrm{NH}), 7.55-7.10(\mathrm{~m}, 13 \mathrm{H}$, Ar-H), 3.87 (s, $\left.3 \mathrm{H}, \mathrm{OCH}_{3}\right), 3.82\left(\mathrm{~s}, 3 \mathrm{H}, \mathrm{OCH}_{3}\right) \mathrm{ppm}$. Anal. Calcd for $\mathrm{C}_{23} \mathrm{H}_{20} \mathrm{~N}_{2} \mathrm{O}_{2}: \mathrm{C}, 77.51 ; \mathrm{H}, 5.66 ; \mathrm{N}$, 7.86; found: C, 77.50; H, 5.64; N, 7.83\%.

\section{2-(2-Chloro-6-fluorophenyl)-4,5-diphenyl-1 $\mathrm{H}$-imida-}

zole (4o). A pale brown solid, $309 \mathrm{mg}$ ( $89 \%$ yield). IR $\left(\right.$ ATR, $\left.\mathrm{cm}^{-1}\right)$ : $3325(\mathrm{NH})$; mp $263-265^{\circ} \mathrm{C} .{ }^{1} \mathrm{H}$ NMR (DMSO- $d_{6}, 400 \mathrm{MHz}$ ): $\delta 12.81$ (s, 1H, NH), 7.61-7.24 $(\mathrm{m}, \quad 13 \mathrm{H}, \quad \mathrm{Ar}-\mathrm{H}) \quad \mathrm{ppm} .{ }^{13} \mathrm{C}$ NMR (DMSO- $d_{6}$, $100 \mathrm{MHz}$ ): $\delta 136.9,134.9,134.6,134.5,131.8,131.7$, $130.7,129.7,129.6,129.2,129.0,128.7,128.4,128.1$, 128.0, 127.8 (2C), 127.7, 127.1, 126.5, 125.7 ppm. ESIMS: $[\mathrm{M}+\mathrm{H}]$ 349.3. Anal. Calcd for $\mathrm{C}_{21} \mathrm{H}_{14} \mathrm{~N}_{2} \mathrm{ClF}$ : $\mathrm{C}$, 72.31; H, 4.05; N, 8.03; found: $\mathrm{C}, 72.30 ; \mathrm{H}$, $4.03 ; \mathrm{N}, 8.02 \%$.

\section{ACKNOWLEDGMENT}

This work was financially supported by the Council of Scientific and Industrial Research (CSIR), New Delhi, India (www.csirhrdg.res.in; no. 02(0172)/ 13/EMR-II dated: Oct. 21, 2013; May 29, 2014; and Oct. 19, 2016).

\section{Supporting information}

Additional supporting information is available in the online version of this article.

\section{REFERENCES}

1. S. Pal, M. N. Khan, S. Karamthulla, L. H. Choudhury, RSC Adv. 2013, 3, 15705.

2. D. Shamala, K. Shivashankar, Chandra, M. Mahendra, Synth. Commun. 2016, 46, 433.

3. K. B. Puttaraju, K. Shivashankar, $R S C A d v$. 2013, 3, 20883.

4. K. B. Puttaraju, K. Shivashankar, Chandra, M. Mahendra, V. P. Rasal, P. N. V. Vivek, K. Rai, M. B. Chanu, Eur. J. Med. Chem. 2013, 69, 316.

5. R. Ghosh, B. De, Int. J. Pharm. Sci. Rev. Res. 2013, 23, 237.

6. V. Gupta, V. Kant, Sci. Int. 2013, 253.

7. M. A. Iradyan, N. S. Iradyan, F. G. Arsenyan, G. M. Stepanyan, Pharm. Chem. J. 2009, 43, 439.

8. A. Bhatnagar, P. K. Sharma, N. Kumar, Int. J. PharmTech Res. 2011, 3, 268.

9. K. Shalini, P. K. Sharma, N. Kumar, Der Chemica Sinica 2010, $1,36$.

10. S. Khaksar, S. Alipour, Monatsh. Chem. 2013, 144, 395.

11. S. L. Gaonkar, K. M. L. Rai, N. S. Shetty, Med. Chem. Res. 2009, 18, 221.

12. A. Karakurt, S. Dalkara, M. Ozalp, S. Ozbey, E. Kendi, J. P. Stables, Eur. J. Med. Chem. 2001, 36, 421.

13. Y. Ozkay, I. Isikdag, Z. Incesu, G. Akalin, Eur. J. Med. Chem. 2010, 45, 3320.

14. J. Pandey, V. K. Tiwari, S. S. Verma, V. Chaturvedi, S. Bhatnagar, S. Sinha, A. N. Gaikwad, R. P. Tripathi, Eur. J. Med. Chem. 2009, 44, 3350.

15. A. Manabe, H. Takano, K. Furuzawa, K. Yanagi, Y. Hisada, S. Tanaka, Biosci. Biotechnol. Biochem. 2002, 66, 2243.

16. S. Lupsor, F. Aonofriesei, M. Iovu, Med. Chem. Res. 2012, 21, 3035.

17. D. Sharma, B. Narasimhan, P. Kumar, V. Judge, R. Narang, E. D. Clercq, J. Balzarini, Eur. J. Med. Chem. 2009, 44, 2347.

18. B. F. A. Wahab, G. E. A. Awad, F. A. Badria, Eur. J. Med. Chem. 2011, 46, 1505.

19. E. H. Nunez, H. Tlahuext, R. M. Puc, H. T. Gomez, R. R. Martinez, R. C. Rivera, C. N. Zuazo, G. N. Vazquez, Eur. J. Med. Chem. 2009, 44, 2975. 
20. N. Rani, A. Sharma, R. Singh, Mini-Rev. Org. Chem. 2015, 12, 34.

21. B. Ashish, S. N. Pandeya, Int. J. Res. Ayurveda Pharm. 2011, 2, 1124.

22. A. Chawla, A. Sharma, A. K. Sharma, Der Pharma Chem. 2012, 4, 116.

23. D. Nagargoje, P. Mandhane, S. Shingote, P. Badadhe, C. Gill, Ultrason. Sonochem. 2012, 19, 94.

24. C. Mukhopadhyay, P. K. Tapaswi, Green Chem. Lett. Rev. 2012, 5, 109.

25. J. J. Garcia, P. Z. Silva, G. R. Rios, M. G. Crestani, A. Arevalo, R. B. Francisco, Chem. Commun. 2011, 47, 10121.

26. M. G. Shen, C. Cai, W. B. Yi, J. Fluorine Chem. 2008 , 129, 541.

27. K. Sivakumar, A. Kathirvel, A. Lalitha, Tetrahedron Lett. 2010, 51, 3018.

28. R. S. Joshi, P. G. Mandhane, M. U. Shaikh, R. P. Kale, G. H. Gill, Chin. Chem. Lett. 2010, 21, 429.

29. J. N. Sangshetti, N. D. Kokare, S. A. Kotharkar, D. B. Shinde, Chin. Chem. Lett. 2008, 19, 762.

30. A. R. Khosropour, Ultrason. Sonochem. 2008, 15,659 .

31. M. M. Heravi, K. Bakhtiari, H. A. Oskooie, S. Taheri, J. Mol. Catal. A Chem. 2007, 263, 279.

32. K. Vikrant, M. Ritu, S. Neha, Res. J. Chem. Sci. 2012, 2, 18.

33. J. Safari, S. D. Khalili, S. H. Banitaba, Synth. Commun. 2011, 41, 2359.

34. M. M. Heravi, M. Zakeri, H. Haghi, Synth. React. Inorg. Met.-Org., Nano-Met. Chem. 2011, 41, 1310.

35. G. V. M. Sharma, M. Jyothi, S. P. Lakshmi, Synth. Commun. 2006, 36, 2991.

36. J. N. Sangshetti, N. D. Kokare, S. A. Kotharkar, D. B. Shinde, Monatsh. Chem. 2008, 139, 125.

37. L. Wang, C. Cai, Monatsh. Chem. 2009, 140, 541.

38. A. A. Mohammadi, M. Mivechi, H. Kefayati, Monatsh. Chem. 2008, 139, 935.

39. K. Bahrami, M. M. Khodaei, A. Nejati, Monatsh. Chem. 2011, 142, 159.

40. S. F. Hojati, S. A. Nezhadhoseiny, Z. Beykzadeh, Monatsh. Chem. 2013, 144, 387.
41. C. Kurumurthy, G. S. Kumar, G. M. Reddy, P. Nagender, P. S. Rao, B. Narsaiah, Res. Chem. Intermed. 2012, 38, 359.

42. J. N. Sangshetti, N. D. Kokare, S. A. Kotharkara, D. B. Shinde, J. Chem. Sci. 2008, 120, 463.

43. P. D. Sanasi, D. Santhipriya, Y. Ramesh, M. R. Kumar, B. Swathi, K. J. Rao, J. Chem. Sci. 2014, 126, 1715.

44. J. Safari, S. D. Khalili, S. H. Banitaba, J. Chem. Sci. 2010, 122, 437.

45. S. S. Pandit, S. K. Bhalerao, U. S. Aher, G. L. Adhav, V. U. Pandit, J. Chem. Sci. 2011, 123, 421.

46. A. Mohammadi, H. Keshvari, R. Sandaroos, H. Rouhi, Z. Sepehr, J. Chem. Sci. 2012, 124, 717.

47. L. Poorali, B. Karami, K. Eskandari, M. Azizi, J. Chem. Sci. 2013, 125, 591.

48. J. Safari, S. G. Ravandi, S. Naseh, J. Chem. Sci. 2013, $125,827$.

49. J. Safari, Z. Zarnegar, J. Chem. Sci. 2013, 125, 835.

50. M. Hajjami, A. G. Choghamarani, Z. Yousofvand, M. Norouzi, J. Chem. Sci. 2015, 127, 1221.

51. A. Garima, V. P. Srivastava, L. D. S. Yadav, Tetrahedron Lett. 2010, 51, 6436.

52. D. Shamala, K. Shivashankar, Synth. Commun. 2016, 46, 1735 .

53. D. Shamala, K. Shivashankar, Synth. Commun. 2017, 47, 105.

54. M. Beerappa, K. Shivashankar, Synth. Commun. 2016, 46, 421 .

55. M. Beerappa, K. Shivashankar, $R S C A d v$. 2015, 5, 30364.

56. N. Jagadishbabu, K. Shivashankar, J. Heterocycl. Chem. doi: $10.1002 /$ jhet. 2742 .

57. N. Jagadishbabu, K. Shivashankar, RSC $A d v$. 2015, 5, 95240.

58. V. S. V. Satyanarayana, A. Sivakumar, Chem. Pap. 2011, $65,519$.

59. F. Xu, N. Wang, Y. Tian, G. Li, J. Heterocycl. Chem. 2013, 50, 668 .

60. R. Wang, C. Liu, G. Luo, Green Chem. Lett. Rev. 2010, 3, 101 .

61. K. F. Shelke, S. B. Sapkal, G. K. Kakade, B. B. Shingate, M. S. Shingare, Green Chem. Lett. Rev. 2010, 3, 27. 